\title{
Influenza vaccine in dialysis patients—time for a reappraisal
}

The influenza vaccine is recommended for patients on dialysis, but data regarding the effectiveness of the vaccine in this population are sparse. In order to assess vaccine effectiveness, Leah McGrath and colleagues used information from the US Renal Data System to compare vaccine effectiveness rates between patients who were vaccinated in 1998, 1999 and 2001 (years in which the vaccine matched the circulating influenza strain) and patients vaccinated in 1997 (a mismatched year). "By utilizing this natural experiment, we could eliminate bias inherent in traditional effectiveness studies of the influenza vaccine", say McGrath and Alan Brookhart from the University of North Carolina.

The researchers examined the outcomes all-cause mortality, hospitalization associated with influenza or pneumonia, and influenza-like illness. More than 100,000 individuals on hemodialysis were included from each year, with vaccination rates being approximately $48 \%$. The researchers first carried out traditional analyses comparing vaccinated and unvaccinated patients, and found strong evidence of confounding bias, even after adjusting for many clinical factors. In addition, the number of events was higher in vaccinated patients in all matched years than in vaccinated patients in the mismatched year, and there was little difference in the survival curves for each outcome. "There are two major findings

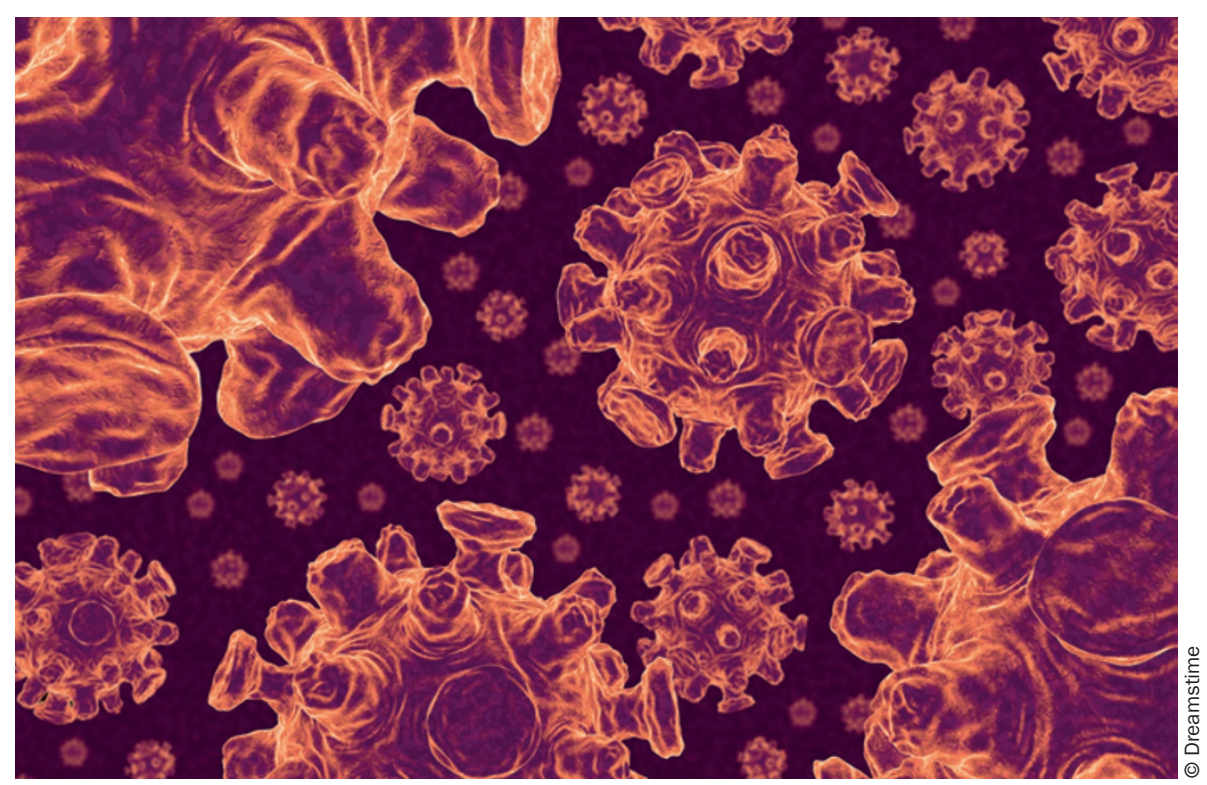

of our study", say McGrath and Brookhart.

"First, the influenza vaccine likely does not prevent a large proportion of wintertime deaths, hospitalizations related to influenza or pneumonia, or influenzalike illness in patients on dialysis. Second, analyses from observational studies that compare vaccinated patients with unvaccinated patients are confounded by hard to measure patient-level behaviors, which can lead to spurious, large protective effects of the vaccine."

"We hope that the results will encourage discussion among scientists and policy makers to develop a better vaccination strategy for patients on dialysis", say the researchers. "Alternative vaccines or high dose vaccines may be more effective in dialysis patients, but we should also remember the value standard prevention measures, such as regular hand washing and avoiding contact with infected individuals."

Helene Myrvang

Original article McGrath, L. J. et al. Influenza vaccine effectiveness in patients on hemodialysis: an analysis of a natural experiment. Arch. Intern. Med. 172, 548-554 (2012) 